\title{
La confianza en el transporte público en relación con la oferta, la demanda y la percepción de la calidad del servicio
}

\author{
Antonio Sanz Bondía \\ Ingeniero Técnico de Obras Públicas, Ingeniero Civil, Máster en Ferrocarriles \\ y Transporte Ferroviario, y Licenciado en Ciencias Ambientales. \\ Jefe de Estudios, Vialobra, S.L., Valencia, España.
}

\section{RESUMEN}

Las grandes ciudades presentan importantes problemas de congestión y contaminación. Desplazar la demanda del vehículo privado hacia modos más sostenibles pertenecientes a las redes de transporte público es una tarea compleja, pero factible mediante criterios técnicos, económicos, medioambientales y de calidad, tanto desde el punto de vista de los operadores como de los usuarios.

La posibilidad de que la ciudadanía valore las ventajas del transporte colectivo, en comparación con las características del vehículo privado, precisa un incremento de la confianza en el primero, desarrollando políticas conjuntas en los distintos modos que aumenten y aproximen la calidad ofertada a la calidad percibida por los clientes.

Esa confianza de la población se fundamenta en conceptos diversos como una planificación adecuada de la oferta ajustada a la demanda; una estructura organizativa capaz de recuperar la eficiencia en caso de incidencias; un mantenimiento continuo de las infraestructuras, instalaciones y material móvil; y una correcta gestión de la información a los usuarios mediante los medios tradicionales y las nuevas tecnologías.

\section{MOVILIDAD URBANA}

Las grandes ciudades y áreas metropolitanas presentan importantes problemas de congestión y contaminación atmosférica y acústica, por el elevado tráfico motorizado interno y externo.

Los motivos de un desplazamiento pueden ser muy diversos: por trabajo, estudios, gestiones administrativas, médico, compras, ocio o asuntos personales, que pueden ser efectuados a pie, en bicicleta, en transporte público o en vehículo privado.

Los diferentes modos de transporte podemos diferenciarlos o agruparlos según ciertas características generalistas:

- Transporte urbano e interurbano.

- Transporte motorizado y no motorizado.

- Uso privado/personal o público/compartido. 
Desde la vertiente económica, los costes varían según el modo de transporte: combustibles, electricidad...

Si elegimos una visión medioambiental, la congestión del tráfico tiene una influencia directa sobre la contaminación atmosférica y acústica, incrementando los consumos energéticos y contribuyendo al cambio climático con la emisión de gases de efecto invernadero.

El aumento de la densidad de la circulación termina afectando a la regularidad del transporte público, lo que hace perder la confianza en él de sus usuarios, que pueden pasar de forma temporal o definitiva al vehículo privado. Con ello se ocupa cada vez más espacio, manteniéndose la congestión habitual en horas punta, y aumentando también el número de vehículos en horas valle. Así se completa un círculo vicioso sobre el que las instituciones deben actuar y en el que la población se ha de concienciar.

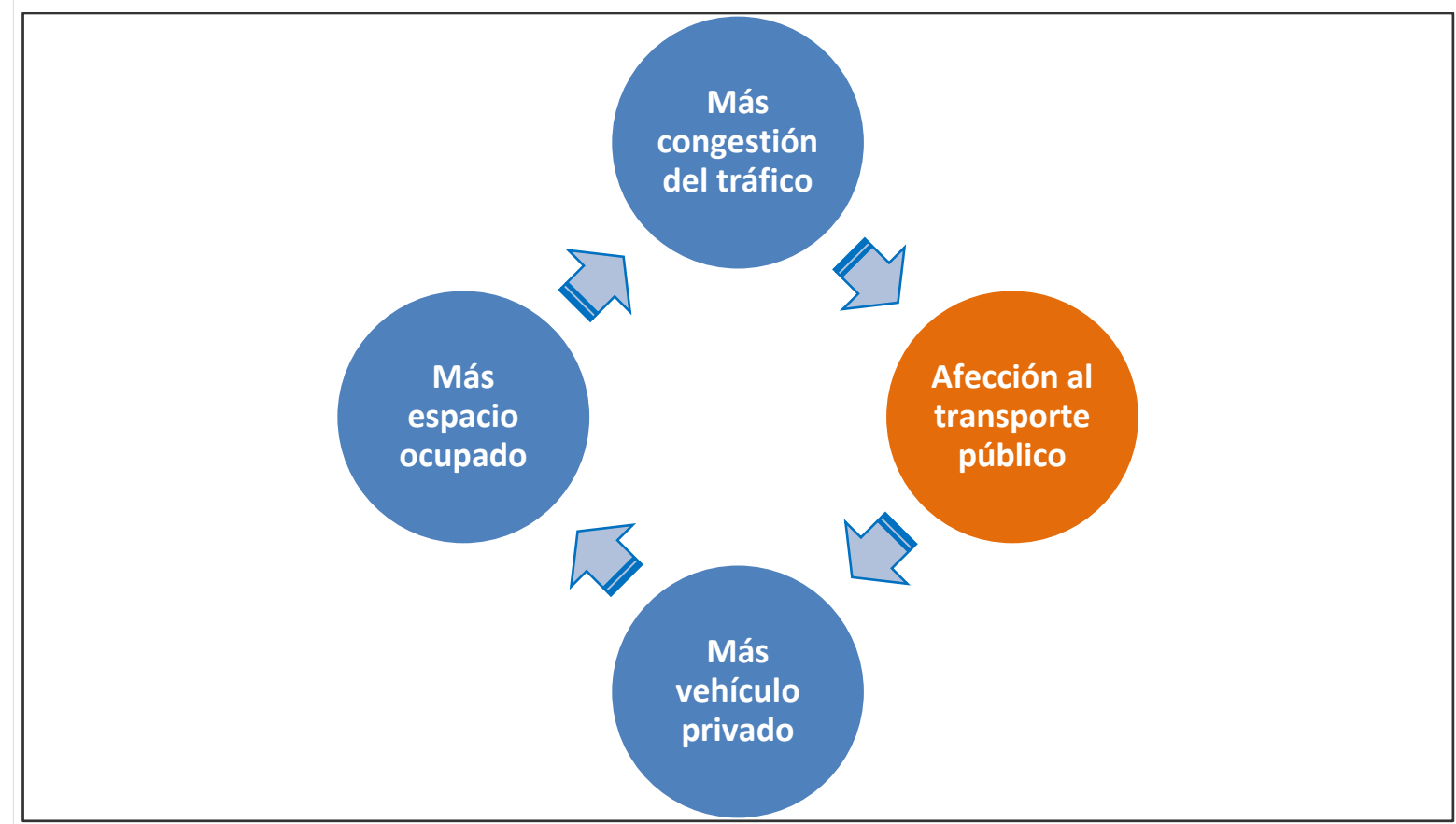

Fig. 1 - Gráfico del círculo vicioso del transporte urbano.

A partir de la evolución del concepto tradicional de tráfico, la movilidad urbana sostenible se define como el conjunto de procesos y acciones orientados al desplazamiento de personas y mercancías, en un entorno urbano, con un coste económico razonable, minimizando los efectos negativos sobre el entorno y la calidad de vida de las personas.

Conseguir desplazar la demanda del vehículo privado hacia modos más sostenibles pertenecientes a las redes de transporte público es una tarea compleja, pero abordable aplicando criterios técnicos, económicos, medioambientales y de calidad, tanto desde el punto de vista de los operadores y los encargados de la explotación, como de los usuarios potenciales. 


\section{OFERTA, DEMANDA Y CALIDAD}

El equilibrio entre cantidad de plazas ofertadas por el operador y número de viajes demandados por los clientes, es un problema difícil de ajustar matemáticamente.

El objetivo de la oferta es pues satisfacer la demanda, con la adaptación y redistribución de los medios personales y materiales de las empresas de transporte en función de las circunstancias de cualquier periodo temporal:

- Días laborables.

- Días festivos.

- Días intermedios, es decir, específicos con previsiones de demanda situadas entre las cifras de una jornada laborable y un festivo.

- Eventos especiales: deportivos, musicales, culturales, festivos...

La posibilidad de que la ciudadanía valore las ventajas del transporte colectivo -en cuanto a horarios, regularidad, costes, emisiones contaminantes o calidad del servicio- en comparación con las del vehículo privado, exige un incremento de la confianza en el público, mediante el desarrollo de políticas que aumenten y aproximen la calidad ofertada por las redes de transporte a la calidad percibida por los clientes.

Una oferta competitiva frente a otros modos alternativos es aquella que cree en una mejora continua en la prestación del servicio y busca una percepción positiva del mismo por parte de los usuarios, orientando el servicio a la satisfacción del cliente en todas sus etapas de viaje.

La certificación de los servicios de transporte público de pasajeros mediante la norma UNE-EN 13816 se basa en la asunción de compromisos por parte de los operadores en cuanto a confort, información, accesibilidad, seguridad, servicio ofertado, atención al cliente e impacto ambiental.

Esta norma es aplicable por tanto al autobús urbano e interurbano, así como al metro, ferrocarril de cercanías, tranvía y metro ligero, y es compatible con la certificación de otros sistemas de Gestión de la Calidad (UNE-EN 9001), Gestión Ambiental (UNE-EN 14001) y Gestión de Seguridad y Salud en el Trabajo (OHSAS 18001) de las empresas de transporte de viajeros.

El ciclo de calidad total es aquel que se va retroalimentando a partir de la calidad esperada por el cliente, de modo que el operador planifica una calidad objetivo, que se convierte en una calidad ofrecida realmente en la explotación, y que será experimentada como una calidad percibida por el usuario durante su etapa de transporte, la cual es comparable con la esperada inicialmente y determinará su confianza en el sistema de transporte y su fidelidad en posteriores usos de la red. 


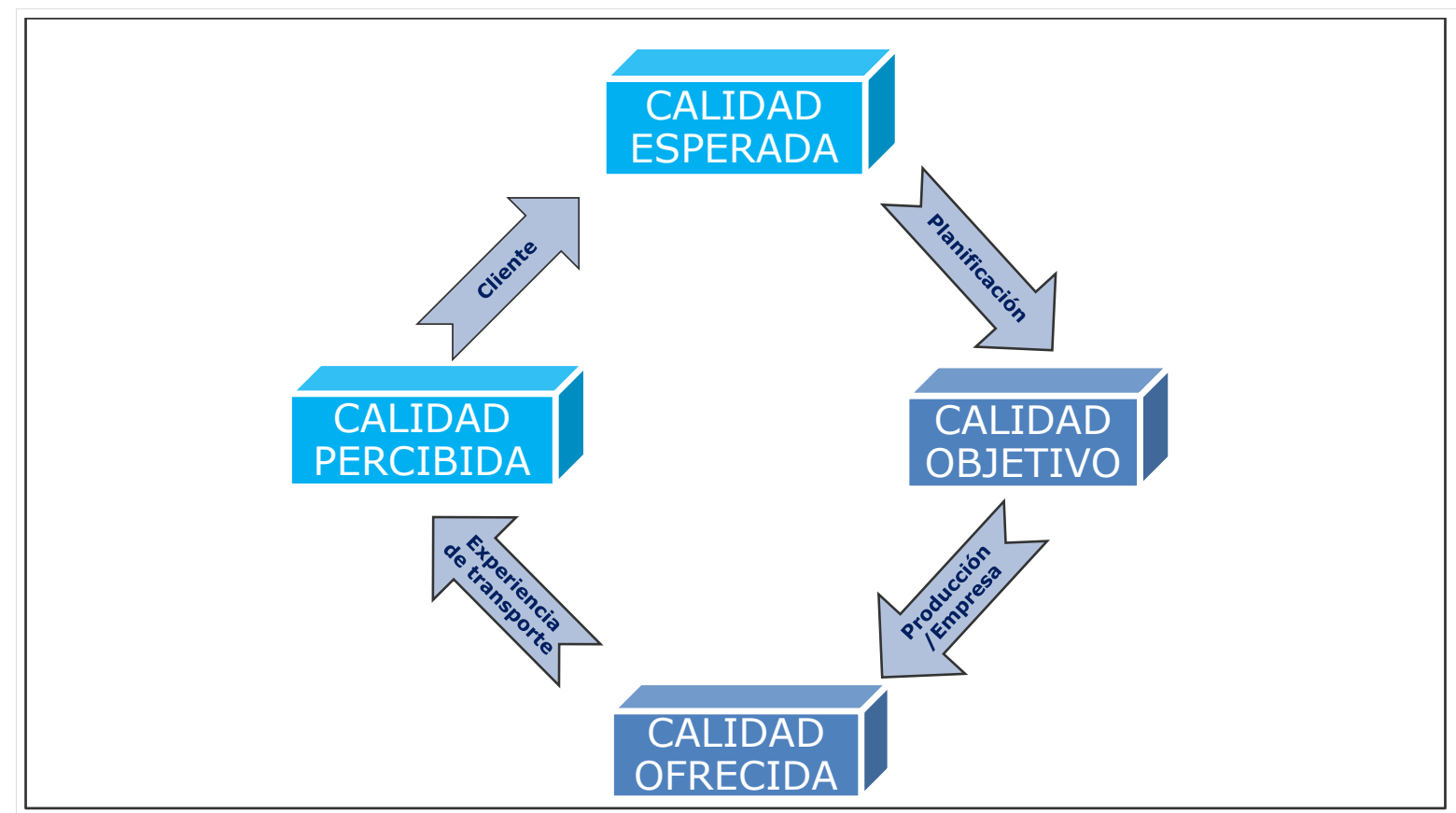

Fig. 2 - Gráfico del ciclo de la calidad total en el transporte público

Esa confianza del cliente potencial está basada en varios conceptos:

- Una planificación adecuada en cuanto a oferta adaptada en lo posible a la demanda.

- Una estructura organizativa capaz de dar respuesta y aplicar las medidas correctoras para la recuperación de la eficiencia en caso de incidencias.

- Un mantenimiento continuo de las infraestructuras, instalaciones y material móvil.

- Una correcta gestión de la información a los usuarios mediante los medios tradicionales y las nuevas tecnologías de la información y la comunicación (TIC), que les permita planificar, de forma previa o inmediata, un desplazamiento ante una necesidad de transporte.

\section{PLANIFICACIÓN}

En cada modo de transporte público destacan algunos conceptos vinculados a la oferta y al objetivo de alcanzar su equilibrio con la demanda variable:

a) En el autobús, metro o tranvía:

- La flota de autobuses o trenes.

- La ruta de cada línea y la ubicación de las paradas.

- La frecuencia de paso en horas punta o valle.

b) En el taxi:

- La flota total de coches en el área de prestación.

- El número de vehículos autorizados para circular en cada jornada y franja horaria. 
c) Y en la bicicleta pública:

- El número de abonados, permanentes o esporádicos, que tan solo aportan una previsión de tendencias de uso.

- La flota de bicicletas.

- El número y ubicación de estaciones en los barrios y en los puntos de atracción y generación de viajes: centro ciudad, universidades y centros educativos, zonas ajardinadas, áreas de relevancia turística...

- El horario de funcionamiento del sistema, con disponibilidad parcial o las 24 horas.

- La redistribución programada o en tiempo real de bicicletas de unas paradas a otras por saturación/vaciado en horas punta, efectuada mediante remolques del concesionario.

- La influencia del clima y las condiciones meteorológicas, que condicionan el número de desplazamientos o la paralización de la flota en una foto fija a partir de una inclemencia, alterando los viajes de ida o vuelta habituales.

Sin embargo, adicionalmente existen otros condicionantes que afectan directamente a las necesidades de movilidad, decantando la elección entre transporte público y privado:

- Empleo: La coyuntura económica condiciona el número de desplazamientos por motivos laborales, evolucionando de forma paralela.

- Consumo: En relación con el empleo y los ingresos, cuando la población reduce gastos también reduce los desplazamientos que pueden considerarse prescindibles.

- Población: Las variaciones demográficas alteran el número de usuarios potenciales de cada modo, lo cual va unido al poder adquisitivo de los clientes ganados o perdidos.

- Hábitos: Los cambios de costumbres por la situación personal modifican los patrones de movilidad individuales; y en su conjunto, los colectivos.

- Disponibilidad de vehículo propio: Los viajeros cautivos no tienen capacidad de elección. El resto puede elegir la opción más cómoda desde un punto de vista individual, independientemente del interés general.

- Tarifas: Cuando estas se incrementan, inicialmente se reduce el número de viajes en transporte público, recuperándose progresivamente si representan una variación de precio razonable.

- Combustibles: El precio del petróleo puede alterar al alza o a la baja la frecuencia de uso de los modos motorizados. Los vehículos eléctricos e híbridos, conforme vayan aumentando su presencia en el parque automovilístico irán modificando la tasa de dependencia respecto a los hidrocarburos.

- Congestión: La velocidad comercial o media de cada tipo de transporte incide en la elección de modo, según el tiempo disponible o que se está dispuesto a asumir para un viaje.

- Intermodalidad: La realización uno o más trasbordos puede penalizar el tiempo de viaje si no hay facilidad o comodidad en los intercambiadores de líneas o de modos. 
- Modificación de la oferta: La implantación o supresión de servicios y líneas, variando la oferta de transporte y los tiempos de espera, hace que el cliente se quede o bien busque otras alternativas a pie o en otros modos.

- Huelgas: Las reivindicaciones laborales en las empresas de transporte público que afectan al servicio de viajeros desplazan puntualmente la demanda hacia el vehículo privado.

Así pues, la oferta y la demanda dependen de muchos factores directos e indirectos.

\section{RECUPERACIÓN DE LA EFICIENCIA POR INCIDENCIAS}

Al igual que, tras la adquisición de un producto, un deficiente servicio postventa puede hacer que un cliente deje de confiar en una marca o en una empresa de reparaciones, una inadecuada respuesta ante una incidencia en una red de transporte público puede hacer perder un cierto número de usuarios, en algunos casos difícil de recuperar en función de su relevancia o su reiteración.

Planteamos a continuación un cuadro de ejemplos de incidencias genéricas, habituales en las flotas de los sistemas de transporte (averías...) o en las infraestructuras por las que discurren (cortes de calles o vías por motivos diversos), y una previsión básica de soluciones alternativas para el mantenimiento provisional del servicio en las mejores condiciones para el cliente hasta su restablecimiento definitivo, y si es posible en un nivel similar al habitual o planificado:

\begin{tabular}{|c|c|c|}
\hline MODO & TIPO DE INCIDENCIA & POSIBLE SOLUCIÓN \\
\hline \multirow[t]{2}{*}{ AUTOBÚS } & En la flota & $\begin{array}{l}\text { Disposición de vehículos y conductores } \\
\text { reserva }\end{array}$ \\
\hline & En el viario & Reordenación temporal de rutas \\
\hline \multirow{2}{*}{$\begin{array}{l}\text { METRO Y } \\
\text { TRANVÍA }\end{array}$} & En la flota & Disposición de trenes y maquinistas reserva \\
\hline & $\begin{array}{l}\text { En la vía, catenaria o } \\
\text { instalaciones }\end{array}$ & $\begin{array}{l}\text { Reajuste de gráficos, supresión parcial del } \\
\text { servicio o servicio sustitutorio de autobuses }\end{array}$ \\
\hline \multirow[b]{2}{*}{ TAXI } & En la flota & Disponibilidad del resto de flota \\
\hline & En el viario & $\begin{array}{l}\text { Ruta alternativa por parte del conductor o } \\
\text { elección de otros modos }\end{array}$ \\
\hline \multirow{2}{*}{$\begin{array}{l}\text { BICICLETA } \\
\text { PÚBLICA }\end{array}$} & En la flota & Uso del resto de unidades \\
\hline & $\begin{array}{l}\text { En el viario o inclemencias } \\
\text { meteorológicas }\end{array}$ & Movilidad peatonal o en otros modos \\
\hline
\end{tabular}

Fig. 3 - Tabla de previsión de incidencias

En todas estas actuaciones resulta de vital importancia contar con unas adecuadas estructuras físicas y humanas de los Puestos de Mando, Centros de Control y sus correspondientes Sistemas de Ayuda a la Explotación (SAE), que permitan gestionar de forma ágil cualquier imprevisto. 


\section{MANTENIMIENTO DE INFRAESTRUCTURAS, INSTALACIONES Y MATERIAL MÓVIL}

Una forma adecuada de mantener la calidad del servicio y generar satisfacción en el cliente es el correcto mantenimiento de los medios disponibles, si bien algunas actuaciones van más allá de las propias empresas de transporte y han de ser llevadas a cabo necesariamente por otras administraciones competentes:

a) Se requiere un adecuado mantenimiento de las infraestructuras:

- Calzadas.

- Aceras.

- Carriles bici y ciclo-calles.

- Vías: infraestructura y superestructura de vía, catenaria e instalaciones de seguridad y comunicaciones.

- Instalaciones fijas: paradas, marquesinas, estaciones, andenes.

b) Se necesitan instalaciones y equipamientos para el mantenimiento del material móvil:

- Cocheras.

- Talleres.

- Equipos móviles de asistencia en ruta.

c) Se ha de atender al mantenimiento regular del material móvil:

- Mecánica.

- Limpieza.

- Confort para el usuario, con especial atención a su adaptación para personas con discapacidad, posibilitando con ello el uso accesible para la totalidad de la población.

- Renovación de vehículos, disminuyendo la edad media de cada flota.

\section{GESTIÓN DE LA INFORMACIÓN}

En una época en la que el acceso a la información está literalmente al alcance de la mano mediante los dispositivos móviles, la población espera una respuesta inmediata, en tiempo real, de cuándo va a llegar el próximo autobús, del estado del tráfico, de la disponibilidad de bicicletas públicas en una parada o de los horarios de una línea de ferrocarril.

El usuario exige simplicidad en las modalidades de títulos de transporte y en los medios de pago:

- Billete sencillo: de uso inmediato.

- Abono prepago: para un número determinado de viajes. 
- Abono temporal: durante su periodo de validez, generalmente mensual o anual.

- Tarjeta monedero: con un saldo disponible que va descontando el importe de cada viaje realizado.

- Dispositivos móviles: mediante aplicaciones o tarjetas SIM NFC.

- Red de venta: física (centros de atención al cliente, taquillas, máquinas expendedoras o quioscos), en ruta (por personal de supervisión) o virtual (por internet y aplicaciones móviles).

Tanto en la explotación normal, y sobre todo en circunstancias especiales por incidencias, el cliente ha de ser informado correctamente, ya que la sensación de falta de consideración o incluso de abandono por parte de la empresa operadora puede generar situaciones de molestia, nerviosismo y formulación de quejas al personal más inmediato -sea o no responsable- $\mathrm{o}$ ante los servicios de atención al cliente.

Así pues, los operadores han de tener capacidad de aportar información rápida a través de distintos medios o soportes propios:

- Comunicación directa con los usuarios.

- Servicio de atención al cliente personal o telefónica.

- Cartelería.

- Megafonía.

- Teleindicadores.

- Pantallas de máquinas de venta de títulos.

- Página web corporativa.

- Aplicaciones móviles.

- Redes sociales apropiadas y de relevancia en cada momento: tipo Facebook, Twitter, WhatsApp...

Además se debe seguir un protocolo de comunicación para que los datos aportados al usuario no resulten insuficientes ni contradictorios en cada uno de los medios empleados, de forma que todos los usuarios informados dispongan de los mismos datos.

También se puede considerar el uso o la participación en la difusión de información de otros medios externos a la empresa:

- Radio.

- Televisión.

- Prensa escrita.

- Prensa en red.

- Administraciones públicas. 


\section{CONCLUSIÓN}

Cada red de transporte público tiene sus características singulares que la hacen única respecto a otras similares.

De forma general, los operadores, además de intentar adaptar la oferta de transporte a la demanda, han de aportar adicionalmente una fiabilidad y una calidad global del servicio que pueda ser percibida y valorada por el cliente, generando una confianza y una fidelización al sistema cada vez que se presente una necesidad de movilidad.

\section{REFERENCIAS}

AENOR - ASOCIACIÓN ESPAÑOLA DE NORMALIZACIÓN Y CERTIFICACIÓN (2003). Norma UNE-EN 13816. Transporte. Logística y servicios. Transporte público de pasajeros. Definición de la calidad del servicio, objetivos y mediciones.

ZAMORANO, C., BIGAS, J.M. y SASTRE GONZÁLEZ, J. (2004). Manual para la planificación, financiación e implantación de sistemas de transporte urbano. Consorcio Regional de Transportes de Madrid, Madrid.

PARICIO, J. (2013). ¿Por qué pierde usuarios el transporte público? www.ecomovilidad.net.

SANZ, A. (2014). Ciudades accesibles. Accesibilidad en la movilidad urbana. Cimbra, Revista del Colegio de Ingenieros Técnicos de Obras Públicas e Ingenieros Civiles, N. ${ }^{\circ} 404$, pp. 42-45.

SANZ, A. (2015). Efectos ambientales generales del transporte sostenible en la movilidad urbana. Aplicación a la ciudad de Valencia. Universidad Politécnica de Valencia, Valencia. 